\title{
VOLTAGE CONTROLLED OSCILLATORS
}

\author{
By \\ Jeff Wilkin \\ University of Toronto
}

November 12, 2001 


\section{$\underline{\text { Introduction }}$}

A key circuit used in modern communication systems is the voltage controlled oscillator (VCO). The VCO's output is an AC waveform whose frequency is dependent upon the input voltage. In today's wireless communication systems, a greater maximum frequency range is required by VCOs. With respect to the digital phones that use these circuits, low power consumption, small size and low fabrication costs are important design factors.

This report is an introduction to voltage controlled oscillators. Two styles of VCO (the LC oscillator and the RC relaxation oscillator) are described as well as the factors that influence their design and selection, the recent advances in the design of these circuits, and the next probable advance of these circuits in the state of the art.

\section{$\underline{\text { Background }}$}

A RC relaxation oscillator consists of two cross coupled transistors with their emitters connected to two ends of a capacitor. Figure 1 shows a bipolar version of the oscillator [1]. Q3 and Q4 are emitter follower buffers. The emitters of Q1 and Q2 are connected to voltage controlled current sources (normally identical bipolar transistors Q7 and Q8). When Q1 is on, its collector voltage holds Q2 off and the emitter current of Q1 charges the capacitor. When the emitter voltage of Q1 rises above two Vbe drops from Vcc Q1

turns off, Q2 turns on and the emitter current of Q2 charges the capacitor. The amount of current that flows into the capacitor is dependent upon the voltage controlled current 
source (the collector currents of Q7 and Q8). The frequency at which the capacitor voltage oscillates is given by:

$$
\text { Fosc }=\mathrm{I}(\mathrm{Vin}) /(4 * \text { Vbe*C) }
$$

I(Vin) is the current from the voltage controlled current source. Vbe is known as a swing voltage (the maximum voltage across $\mathrm{C}$ before the transistors switch).

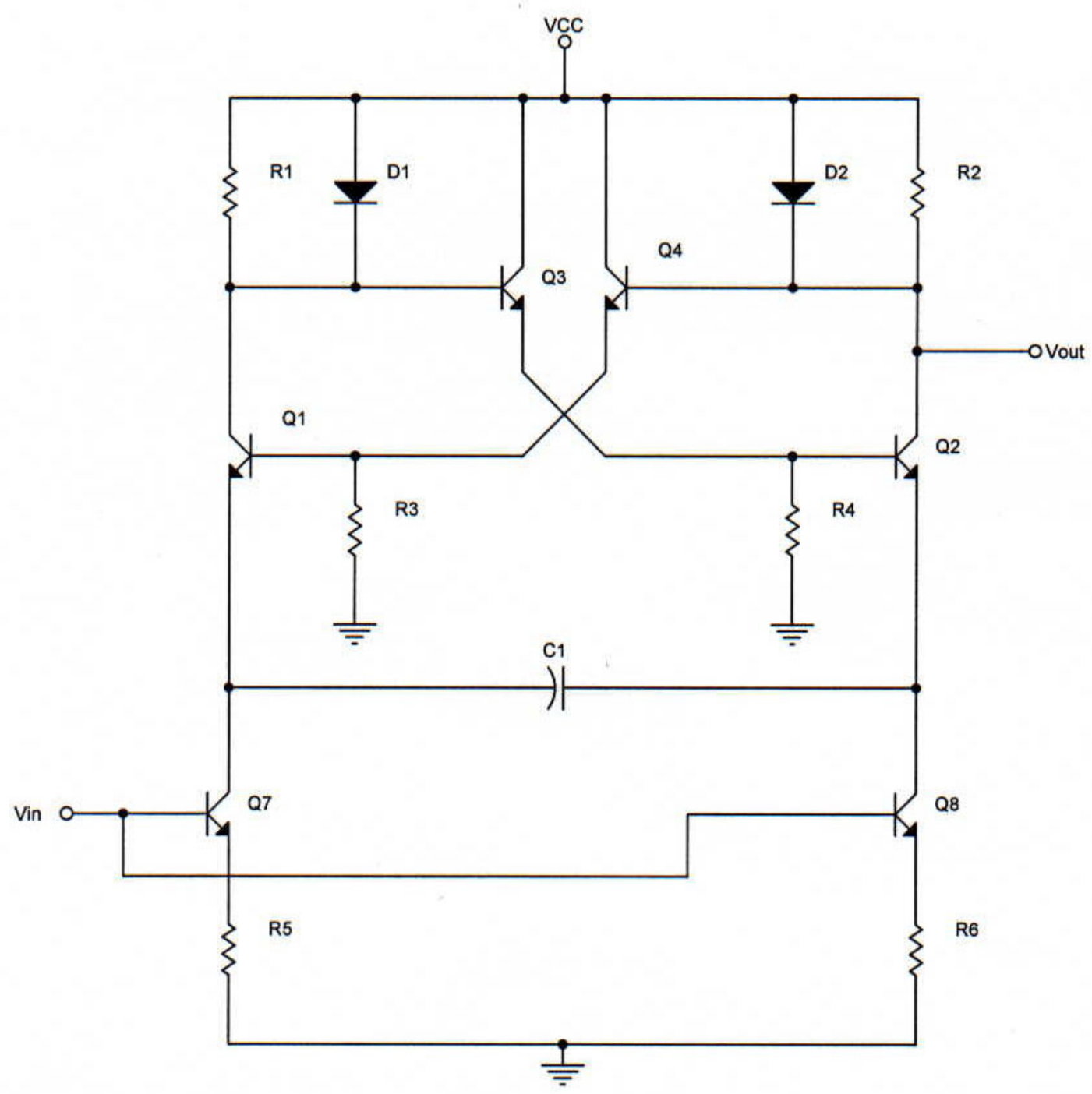

Figure 1: An emitter coupled relaxation voltage controlled oscillator [1].

The active components of the simplest relaxation oscillators consist of four transistors.

The transistors can either be emitter-coupled or collector-coupled. Emitter-coupled 
circuits exhibit sudden voltage jumps at both ends of the capacitor. The voltage jumps create excessive current spikes and possible substrate injection [2]. Sudden voltage jumps do not occur in collector-coupled circuits. Variations of the circuit have used Schmitt triggers or SR latches to drive the capacitor.

The benefits of relaxation oscillators include a large frequency tuning range, a low cost, a very linear frequency versus voltage relationship and a requirement of only a single reactive component. Also, all of the components of this oscillator can be fabricated on a single IC. However, these circuits have poor frequency stability at high frequencies and are more susceptible to phase noise compared to LC oscillators [3]. Also, the relaxation oscillator cannot generate a pure sine wave. If the output is referenced at one of the collector nodes then the measured signal will be a square wave (with peaks between Vcc and ground) whereas the voltage across the capacitor will be a saw-tooth wave with its mean voltage at zero volts.

The output frequency of the relaxation oscillators is also dependent on the circuit temperature. Vbe is inversely proportional to temperature. Therefore as temperature increases the oscillation frequency will increase when the input voltage remains constant.

A LC oscillator generates its AC waveform with the assistance of an inductor-capacitor tank. Feedback to an amplifier is used to help maintain oscillation and to reduce damping. The frequency of the oscillator output is determined by the equation:

$$
\text { Fosc }=1 /(2 \pi \sqrt{ }(\mathrm{LC}))
$$


An input voltage can tune the frequency if a varactor (a semiconductor device with a voltage-dependent capacitance) is used in place of the capacitor. There are several configurations for LC oscillators. The most common are the Hartley, Colpitts and Clapp oscillators (See Fig. 2, 3 and 4. NOTE: In order for these circuits to be voltage controlled, at least one of the capacitors must be replaced with a varactor). The Hartley oscillator is an inductively coupled circuit that has the advantage of having its output amplitude remain constant over the tunable frequency range. However, this circuit generates harmonic frequencies which prevents it from producing pure sine waves.

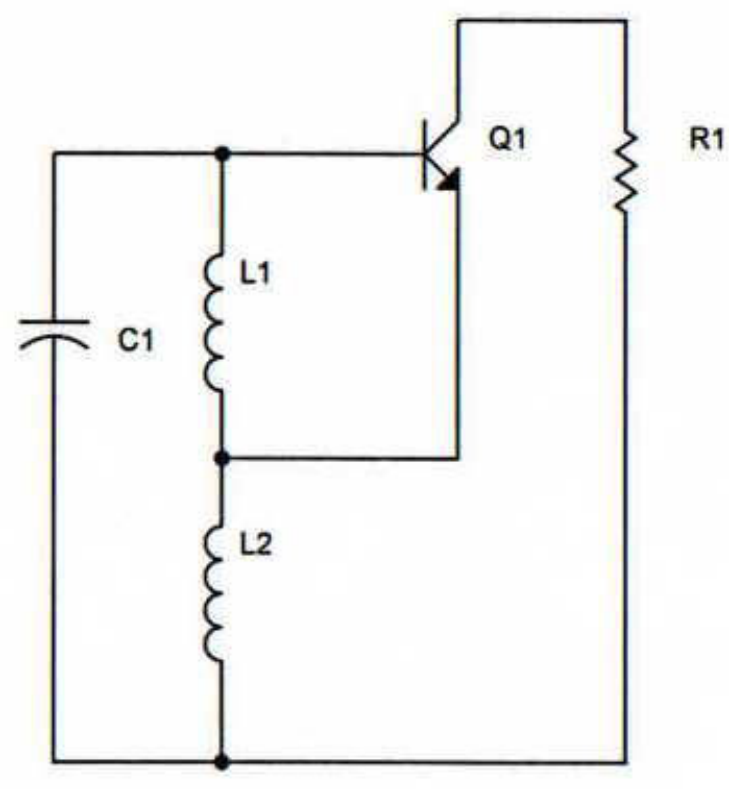

Figure 2: A Hartley LC oscillator [4]

The Colpitts and Clapp oscillators are capacitively coupled circuits that provide better frequency stability than the Hartley oscillator. The Clapp has the added advantage of having a higher loaded Q than the Colpitts. In each design, a transistor is used as a signal amplifier and a resistor is uses as a feedback device [4]. 


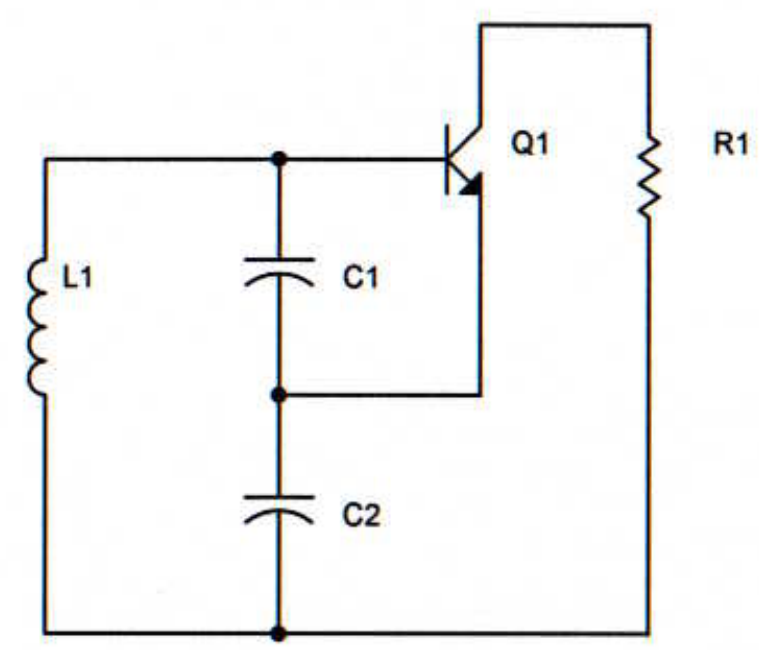

Figure 3: A Colpitts LC oscillator [4]

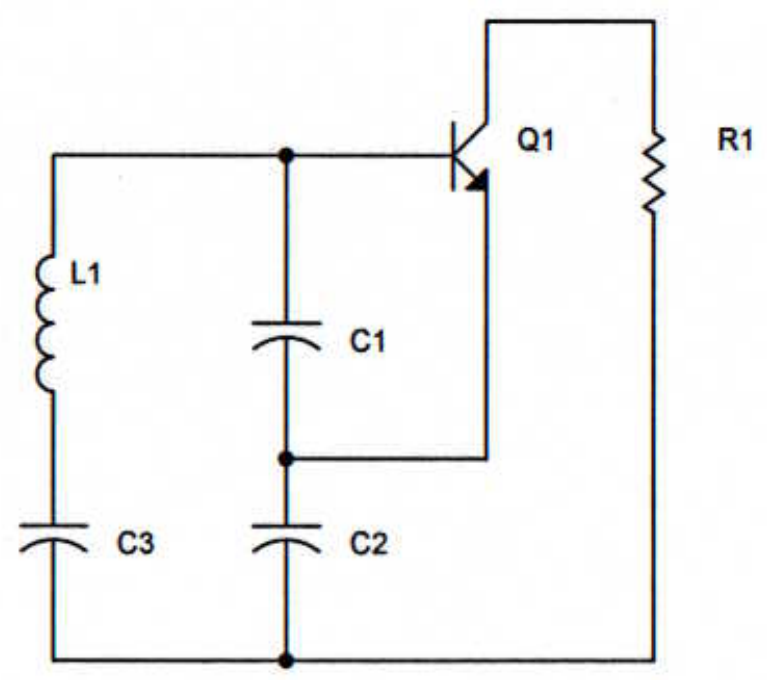

Figure 4: A Clapp LC oscillator [4]

Among the benefits of LC oscillators are high phase stability and low susceptibility to noise. However, traditional LC oscillators have lower tuning range and higher cost compared to relaxation oscillators. 


\section{$\underline{\text { Design Factors }}$}

The design requirements for a VCO are:

- Phase stability

- Large electrical tuning range

- Linearity of frequency versus control voltage

- Large gain factor

- Capability for accepting wideband modulation

- Low cost

Not all of these requirements can be satisfied together [5].

\section{Jitter Noise and Active Components}

The most important design consideration is the effect of noise on the circuit. Relaxation oscillators are subject to fluctuations in the period of their output, known as jitter. Jitter limits the frequency range of the oscillator, timing accuracy and the signal-to-noise ratio (with respect to frequency translation). As the capacitor is charging, the gain of the voltage sensing devices (the switching transistors) is small but becomes "unboundedly large" as the switching threshold is approached. This gain amplifies noise on the bias current thereby causing the capacitor to switch at random times. This causes the output to be a "randomly pulse width modulated waveform". The magnitude of the jitter noise is defined as:

$$
\text { Jitter }=\sigma(\mathrm{T}) / \mu(\mathrm{T})
$$

$\sigma(\mathrm{T})$ and $\mu(\mathrm{T})$ are the standard deviation and the mean of the pulse width respectively [6]. In reference [6] the value of the jitter has been shown to be dependent on the design of the oscillator and can be measured using the following equations:

$$
\sigma(\mathrm{T})=\alpha^{*}(\mathrm{RC} /(\mathrm{Io}-\mathrm{Ir})) \sigma(\mathrm{In})
$$




$$
\sigma(\mathrm{T})=\left(\alpha^{*} \sqrt{ } 6\right)^{*}(\mathrm{Vn}(\mathrm{rms}) / \mathrm{S})
$$

$\alpha$ is a constant dependent upon the slope of the capacitor voltage ramp and the rms noise voltage. $\mathrm{R}$ is the load resistor of the switching transistor. $\mathrm{C}$ is the charging capacitor. Io is the voltage controlled tail current. $\mathrm{Ir}=\mathrm{Vt} / 2 \mathrm{R}$ (where $\mathrm{Vt}$ is the thermal voltage). $\sigma(\mathrm{In})$ is the standard deviation of the noise current. $\mathrm{Vn}(\mathrm{rms})$ is the $\mathrm{rms}$ of the noise voltage. S is the slope of the capacitor voltage at the switching point. It can be seen that increasing tail current Io can minimize jitter noise in a relaxation oscillator. However, this method may conflict with a low power consumption requirement for the circuit.

The active devices used in an oscillator add to the phase noise. The more active components that are used in a circuit, the more susceptible that circuit is to noise. The noise sources in the active devices include their inherent flicker and thermal noise. LC oscillators have an advantage over relaxation oscillator in this respect. The oscillation frequency of the LC circuit is dependent on passive devices (inductors and capacitors) and a single active transistor as opposed to multiple transistors used by the relaxation oscillator.

\section{QFactor}

The quality factor $\mathrm{Q}$ of the oscillator determines the level of damping of the output signal and the susceptibility of the circuit to phase noise. A definition of Q that applies to most oscillators is:

$$
\mathrm{Q}=(\omega 0 / 2)(\mathrm{d} \Phi / \mathrm{d} \omega)
$$

$\omega 0$ is the resonance frequency and $\mathrm{d} \Phi / \mathrm{d} \omega$ is the slope of phase with respect to frequency [10]. A high $\mathrm{Q}$ factor implies that there is a small damping factor and the signal is better 
able to maintain oscillation. If the phase slope is high then a phase shift (as caused by phase noise) in a closed loop system (like a phase lock loop) will force the oscillator to return to the resonance frequency [10]. If the phase slope is low then there is weaker tendency to maintain resonance frequency when phase deviations occur. As a result a high Q helps to make the circuit less susceptible to noise. LC oscillators have a high Q factor by the nature of its design: a good quality inductor and a minimal number of active components. LC oscillators have a $\mathrm{Q}$ factor that is typically four times the $\mathrm{Q}$ factor of a relaxation oscillator [9]. High frequency applications require oscillators with high Q factors. Traditional relaxation oscillators have been limited in their use at high frequencies due to their lower Q factor and subsequent noise susceptibility therefore LC oscillators have been favored. However large Q inductors have to be used off-chip thereby sacrificing size requirements.

\section{Design Improvements}

Early VCOs were constructed with discrete components and were rarely part of integrated circuits. IC oscillators had poor stability and frequency compared to their discrete circuit counterparts [5]. In the last few years, significant developments have been made to improve IC VCO limitations.

\section{$\underline{\text { Relaxation Oscillators }}$}

Early relaxation oscillators had an upper tuning range in the tens of megahertz. Today's circuits have frequency limits in the gigahertz range while still maintaining voltage-tofrequency linearity. This range is made possible with the use of automatic swing control 
(ASC) [2]. When the voltage across the capacitor reaches the switching point, there is a delay before the capacitor starts to charge in the opposite direction. This is due to an inherent delay in the transistors to change states. The delay is dependent upon temperature and process factors. During the delay, the capacitor continues to charge in the same direction. The voltage across the capacitor is no longer fixed at this point and the current-frequency linearity is affected. This limits the maximum frequency that can be reached linearly. The ASC applies a feedback to the VCO that replaces the fixed swing voltage with one that compensates for the swing increase caused by the delay. An applied reference voltage determines the maximum peak of the capacitor voltage. The added feedback stabilizes the peak amplitude allowing the oscillator to achieve a maximum frequency of:

$$
\operatorname{Flim}=1 /(4 * T d)
$$

$\mathrm{Td}$ is the switching delay of the transistors. Results of simulations demonstrated in reference [2] show that, for a given current, oscillation frequency is higher with ASC feedback than without ASC (a 500MHz oscillator without ASC achieved $800 \mathrm{MHz}$ with ASC). The signal amplitude is also more stable and the current-frequency is more linear for higher frequencies. For frequencies below the maximum frequency, the circuit is less sensitive to temperature variations and power supply variations (around a $1 \%$ frequency change for a $5 \%$ power change).

Traditionally, relaxation oscillators using CMOS technology have been used for low frequency applications, but submicron MOS processes have allowed CMOS oscillators to achieve frequencies in the gigahertz range. In order to achieve a higher quality factor, CMOS relaxation oscillators have been designed in the form of ring oscillators. A ring 
oscillator consists of a number of CMOS delay cells (either single ended or differential inverters) connected in series. The output signal of the $\mathrm{n}^{\text {th }}$ cell is connected to the input of the $1^{\text {st }}$ cell for feedback (See Fig. 5). The frequency of the ring oscillator is determined by:

$$
\mathrm{f}=1 /(2 * \mathrm{n} * \text { Tdelay })
$$

Tdelay is the switching delay of a single cell and $\mathrm{n}$ is the total number of cells in the circuit. The use of multiple inverter integrators provides a higher $\mathrm{Q}$ than for a single stage relaxation oscillator [9]. It was demonstrated in reference [9] that CMOS VCOs designed as ring oscillators can achieve higher frequencies and better phase noise performance (comparable to monolithic LC oscillators) than other CMOS VCO designs and lower power consumption than VCOs designed using compatible technologies.

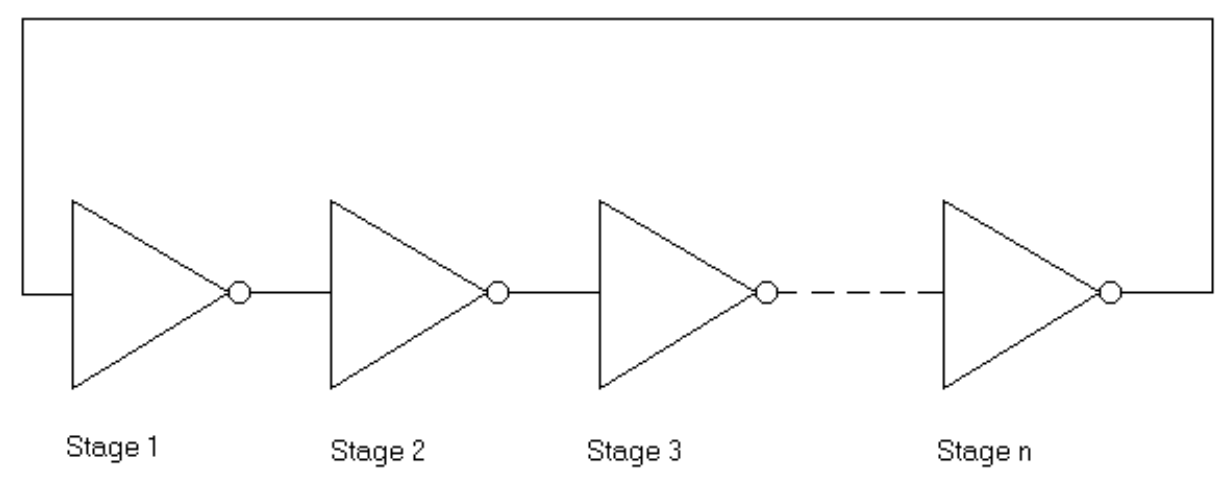

Fig. 5: Block Diagram of a Ring Oscillator.

\section{$\underline{\text { LC Oscillators }}$}

One of the drawbacks of the LC oscillator is that early models could not be fully monolithic since inductors could not be fabricated on the chip. The size of required inductors tended to be too large to fit on ICs therefore they were included as discrete components. One way to by pass this problem is to simulate inductance using active components. This allows the circuit to occupy a single chip, however the addition of the 
active components increases the circuit's susceptibility to phase noise. Larger capacitors and more power are needed to compensate for the noise. Another alternative is to use bondwire. Bondwire has an associated parasitic inductance of around $1 \mathrm{nH}$ per $\mathrm{mm}$ length. This provides finite inductance that can be fabricated on the chip, but great care is needed in fabrication as the bondwires must be kept straight and have a constant separation from each other in order for them to provide the expected inductance [7]. The repeatability of the bondwire technique prevents it from being widely used [8].

Within the last decade, improvements in integrated circuit fabrication have allowed spiral inductors to be fabricated on ICs. The technique involves laying out two metal layers in the form of a rectangle spiral where each layer is on either side of a silicon substrate. The result has been that LC oscillators have become more compact. The quality factor of the LC oscillator is limited by the inductors and therefore it is dependent on the fabrication process. The metal traces have a series resistance that affects the inductor's reactance. Also, the coil has a parasitic capacitance to the substrate that limits its maximum frequency. The combination of added resistance and capacitance places a maximum limit to the achievable inductance, reduces the quality factor and reduces phase stability of the circuit. These problems are less prominent when the circuit is fabricated with a more advanced process technology (such as using etching techniques to remove the silicon substrate) [8]. The available chip area is another limiting factor. For example, if the required inductance demands that the fabricated coils take up an area that does not leave room for the rest of the circuit then the oscillator cannot be fabricated in its entirety on a single chip. 


\section{The Future of VCOs}

As the communications industry evolves, especially in the wireless domain, there will be greater demand for faster data rates, smaller devices and lower power consumption. These demands will push the envelope of VCO design beyond today's limitations. The biggest challenge for designers is to find an optimal balance between these requirements.

Faster switching devices will be necessary to drive oscillators at higher frequencies. Enhanced phase noise reduction circuitry will be needed to ensure the stability of multigigahertz signals without sacrificing low power requirements. As CMOS fabrication techniques continue to advance, relaxation oscillators (designed as ring oscillators) will be able to meet these demands with performance comparable to LC oscillators but with the benefit of being constructed on smaller surface areas.

For LC oscillators, improved fabrication techniques will allow high Q factor inductors to occupy less space on the integrated circuit and will allow the inductance value to be made more repeatable during the manufacturing process. An added benefit will be a wider range of inductor values that can be generated for use "on chip" thereby increasing the tunable frequency range of the LC oscillator. 


\section{$\underline{\text { References }}$}

[1] Grey, R. G. Meyer, “Analysis and Design of Analog Integrated Circuits”, John Wiley \& Sons., 1993.

[2] M. H. Shakiba, T. Sowlati, "Automatic Swing Control in Relaxation Oscillators”, IEEE J. Solid-State Circuits, vol. 33, pp. 1979-1986, December 1998.

[3] N. M. Nguyen, R. G. Meyer, "A 1.8 GHz Monolithic LC Voltage-Controlled Oscillator”, ISSCC 92, pp. 158-159, 1992.

[4] A. Chung, "LC Oscillators: Theory, Design and Trend".

[5] F. M. Gardner, Ph. D, "Phaselock Techniques, $2^{\text {nd }}$ ed.", John Wiley \& Sons, 1979.

[6] A. A. Abidi, R. G. Meyer, "Noise in Relaxation Oscillators", IEEE J. Solid-State Circuits, vol. SC-18, pp. 794-802, December 1983.

[7] J. Craninckx, M. S. J. Steyaert, “ A 1.8-GHz CMOS Low-Phase-Noise VoltageControlled Oscillator with Prescaller”, IEEE J. Solid-State Circuits, vol. 20, pp. 14741482, December 1995.

[8] J. Craninckx, M. S. J. Steyaert, “A 1. 8-GHz Low-Phase-Noise VCO Using Optimized Hollow Spiral Inductors”, IEEE J. Solid-State Circuits, vol. 32, pp. 736744, May 1997.

[9] M. Thamsirianunt, T. A. Kwasniewski, “CMOS VCO’s for PLL Frequency Synthesis in GHz Digital Mobile Radio Communications", IEEE J. Solid-State Circuits, vol. 32, pp. 1511-1524, October 1997.

[10] B. Razavi, "A Study of Phase Noise in CMOS Oscillators", IEEE J. Solid-State Circuits, vol. 31, pp. 331-343, March 1996. 